\title{
Output memory-based event-triggered control
}

\author{
Miguel A. Davó, Mirko Fiacchini and Christophe Prieur
}

\begin{abstract}
This work is concerned with the exponential stability of an output-based control scheme where the measured output is subjected to event-triggered sampling. We propose a new event-based sampling criterion based on the memory of the measured output instead of only the current output. This allows to prevent accumulation of sampling times. The exponential stability is analyzed by using a Lyapunov-based approach, providing a link between the sampling criterion and the decay rate. Several numerical examples illustrate the effectiveness of the proposed event-triggered scheme.
\end{abstract}

\section{INTRODUCTION}

Sampled-data control for continuous-time dynamical systems is a very active research subject. Periodic and aperiodic implementations of nonlinear controllers are now well studied and very good performances can be obtained in terms of resource consumption, of number of sampling times and of the speed of convergence of the closed-loop system, see e.g. [5]. See [11], [12] for recent tutorials on event-triggered techniques for control design.

A common approach for the design of sampled-data control is to first involve a stabilizing feedback controller (and an associate Lyapunov function) and to add a triggering mechanism. Most of the approaches are based on the difference between the current value of the state and the last used state measure, see e.g. [15], [14], [21], [2], [3] and references therein. Some of them assume the existence of an Input-to-State (ISS) Lyapunov function, whereas more recent approaches require less strong assumptions and ask to update the measure of the state only when a Lyapunov function has a sufficiently negative derivative as the solution approaches to the equilibrium (see [18], [19]). Other techniques are based on an observer (or a norm-observer) and require the knowledge of the (sampled) output only, see [22] and [23] respectively for linear and nonlinear control systems. The present work deals with event-triggered mechanisms which depend only on the output (and not on all the state). This yields to an event-triggered output feedback controller when this mechanism is combined with an output feedback controller.

Generalizing event-triggered control techniques, when only the output is known, is definitively non-trivial, since keeping the error (between the last sampled output and the current value of the output) small may lead to Zeno phenomenon [13]. Such bad behavior occurs when we need

M. A. Davó, M. Fiacchini and C. Prieur are with Gipsa-Lab (CNRS), Grenoble, France. This work has been partially supported by the LabEx PERSYVAL-Lab (ANR-11-LABX-0025-01) and by the ANR project LimICoS contract number 12-BS03-005-01. Corresponding author: Miguel.Davo-Navarro@gipsa-lab.fr to send infinite samples in finite-time and hence the eventtriggering mechanism is not feasible for implementation in practice. There are several approaches to avoid Zeno solutions in the closed-loop system, for instance: adding a threshold in the event-triggered mechanisms (leading to practical stability of the origin in the constant case), see e.g. [7], [17], combining time-triggered control and eventtriggered control, see e.g. [10], [1], etc.

Asymptotic stability of the equilibrium is obtained in this paper with an event-triggered controller. More precisely a sequence of triggering times are computed from only the knowledge of the output. At these times, it is necessary to update the output measure used in the controller dynamics. Using only the output requires an observability property written in terms of the distinguishability of the equilibrium of the open-loop control system, as introduced in [20] (see also [4], [6]). Moreover Zeno solutions are avoided. To do that the history of the output is used. The asymptotic stability proof exploits techniques inspired by Lyapunov-Razuminkin theorem, whereas the exponential stability proof is based on the Halanay's inequality (see [9], [8] and references therein, where such techniques have been used for some classes of time-delay systems). When our results are particularized to linear and time-invariant (LTI) control systems, the proposed exponential stability conditions are written in terms of Linear Matrix Inequalities, which may be solved efficiently with Semi-Definite Programming (SDP) solvers.

An important feature of our approach is the possibility of relating the parameters of the sampling algorithm with the speed of convergence. This comes directly from the proof of exponential stability for nonlinear control systems. In addition, several examples suggest that these parameters are also related to the inter-event times, what leads to a classical tradeoff between the speed of convergence and the number of needed updates to achieve this speed of convergence.

This paper is organized as follows. First the problem under consideration is introduced and the event-triggered setup is defined (see Section II). Section III contains the main results on nonlinear control systems. Results are applied and improved in Section IV where we focus on LTI systems. The techniques are applied on a nonlinear control system and a linear one in Section V.

Notation: The sets $\mathbb{S}^{n}$, and $\mathbb{S}_{+}^{n}$ denote the set of symmetric matrices of dimension $n \times n$, and the set of symmetric positive definite matrices of dimension $n \times n$, respectively. The notation $\|x\|$ is the Euclidean norm for $x \in \mathbb{R}^{n}$. The notation $P>0$ for $P \in \mathbb{S}^{n}$ means that $P$ is positive definite $\left(P<0\right.$ means negative definite). For a matrix $A \in \mathbb{R}^{n \times n}$, the notation $H e(A)$ refers to $A+A^{\top}$. For a symmetric matrix 
$A \in \mathbb{R}^{n \times n}, \lambda_{m}(A)$ and $\lambda_{M}(A)$ stand for the minimum and maximum eigenvalue, respectively. For a function $f$ : $[a, b] \rightarrow \mathbb{R}^{n}$, a norm is defined as $\|f\|=\sup _{s \in[a, b]}\|f(s)\|$. A function $f: \mathbb{R}_{+} \rightarrow \mathbb{R}_{+}$is of class $\mathcal{K}$ if it is continuous, strictly increasing, and $f(0)=0$. The function $f$ is of class $\mathcal{K}_{\infty}$ if $f \in \mathcal{K}$, and in addition $\lim _{s \rightarrow \infty} f(s)=\infty$.

\section{Problem statement}

Consider an output-based control system formed by a plant given by

$$
\mathcal{P}:\left\{\begin{array}{l}
\dot{x}_{p}(t)=f_{p}\left(x_{p}(t), u_{p}(t)\right), \\
y_{p}(t)=g_{p}\left(x_{p}(t)\right),
\end{array}\right.
$$

where $x_{p} \in \mathbb{R}^{n_{p}}, u_{p} \in \mathbb{R}^{n_{u}}$, and $y_{p} \in \mathbb{R}^{n_{y}}$ and a feedback controller

$$
\mathcal{C}:\left\{\begin{array}{l}
\dot{x}_{c}(t)=f_{c}\left(x_{c}(t), u_{c}(t)\right), \\
y_{c}(t)=g_{c}\left(x_{c}(t)\right) .
\end{array}\right.
$$

where $x_{c} \in \mathbb{R}^{n_{c}}, u_{c} \in \mathbb{R}^{n_{y}}$, and $y_{c} \in \mathbb{R}^{n_{u}}$. The openloop connection between the controller and the plant with zero input will be denoted by $(\mathcal{C}, \mathcal{P})$, and is defined by $u_{p}(t)=y_{c}(t)$ and $u_{c}(t)=0$. In addition, assume that the feedback interconnection between the plant and the controller is affected by an exogenous signal $e(t) \in \mathbb{R}^{n_{y}}$, such that the interconnection is given by $u_{p}(t)=y_{c}(t)$ and $u_{c}(t)=$ $y_{p}(t)+e(t)$. Considering the state $x=\left[\begin{array}{ll}x_{p}^{\top} & x_{c}^{\top}\end{array}\right]^{\top} \in \mathbb{R}^{n}$ with $n=n_{p}+n_{c}$, the closed-loop system is described by

$$
\left\{\begin{array}{l}
\dot{x}(t)=f(x(t), e(t)) \\
y(t)=g(x(t))
\end{array}\right.
$$

with $g(x(t))=g_{p}\left(x_{p}(t)\right) \in \mathbb{R}^{n_{y}}$ and

$$
f(x(t), e(t))=\left[\begin{array}{l}
f_{p}\left(x_{p}(t), g_{c}\left(x_{c}(t)\right)\right) \\
f_{c}\left(x_{c}(t), g_{p}\left(x_{p}(t)\right)+e(t)\right)
\end{array}\right] .
$$

The function $f$ is assumed to be locally Lipschitz continuous in both arguments and $f(x, e)=0$ if and only if $x=0$ and $e=0$. The function $g(x)$ is assumed to be locally Lipschitz continuous, and in addition, there exists a function $\xi \in \mathcal{K}$ such that $\|g(x)\| \leq \xi(\|x\|)$.

In order to derive the results in this work, the following assumptions are considered:

Assumption 1: Any pair of states $(0, x)$ is distinguishable for the open-loop $(\mathcal{C}, \mathcal{P})$, which means that for any $\varepsilon>0$ if $g(x(t))=0$ for all $t \in[0, \varepsilon)$ where $x(t)$ is a solution to $(\mathcal{C}, \mathcal{P})$, then $x(0)=0$.

Assumption 2: There exists a smooth function $V: \mathbb{R}^{n} \rightarrow$ $\mathbb{R}_{+}$and functions $\underline{\alpha}, \bar{\alpha}, \alpha, \beta \in \mathcal{K}_{\infty}$ such that, for all $x \in \mathbb{R}^{n}$

$$
\begin{gathered}
\alpha(\|x\|) \leq V(x) \leq \bar{\alpha}(\|x\|), \\
\nabla V(x) \cdot f(x, e) \leq-\alpha(\|x\|)+\beta(\|e\|) .
\end{gathered}
$$

Assumption 2 is an Input-to-State property of (3), whereas Assumption 1 is an observability property which requires the state to be zero if the output is zero as well in a time interval.

Consider the closed-loop system formed by the plant (1) and the controller (2), where now the interconnection from the plant to the controller is made through a sensor, that is supposed to have continuous access to the plant. The sensor samples the plant output at some instants $t_{k}, k \in \mathbb{N}$, referred to as sampling times (or triggering times in the context of event-triggered control), and keeps the input of the controller constant until the next sampling time. In this way, the interconnection is given by $u_{p}(t)=y_{c}(t)$ and $u_{c}(t)=y_{p}\left(t_{k}\right)$ for all $t \in\left[t_{k}, t_{k+1}\right), k \in \mathbb{N}$. The sampling times can be generated in a number of ways. In event-triggered control the sampling times are governed by an event-triggered mechanism, that continuously monitors the behavior of the plant and generates events when some condition is satisfied. This work focuses on the emulation-based approach, where first the controller is designed to get some desired behavior for the continuous loop, and second, an event-triggered mechanism is developed to provide a bounded deviation of the eventtriggered implementation from the continuous one, under Assumptions 1 and 2. Therefore, the problem is to design the sampling algorithm, i.e. the computation of the sequence $\left(t_{k}\right), k \in \mathbb{N}$, in order to guarantee the exponential/asymptotic stability of the system and at the same time to prevent Zeno solutions.

Defining the error signal

$$
e(t)=y\left(t_{k}\right)-y(t)
$$

for all $t \in\left[t_{k}, t_{k+1}\right)$ and $k \in \mathbb{N}$, the dynamics of the eventtriggered closed-loop system can be described by (3) and (4) with this particular choice of the error signal $e$.

Due to the space limitation, the proofs are omitted, and are available upon request from the authors.

\section{MAIN RESUlTS}

\section{A. Memory-based event-triggered mechanism}

Let us first extend the initial condition of the system (3) on the interval $[-h, 0]$ as follows: $x(t)=x(0), t \in$ $[-h, 0]$, where $h>0$ is a design parameter of the eventtriggered mechanism. In addition, we define the function $y_{t}:[-h, 0] \rightarrow \mathbb{R}^{n}$, which is given by $y_{t}(s)=y(t+s)$, $s \in[-h, 0]$, where $y(t)$ is the output of the system (3). Beside the parameter $h$, consider two design parameters: a function $\sigma \in \mathcal{K}$ and a time $t_{0}>0$ (it is not a triggering time), then we propose the following sampling algorithm for all $k \in \mathbb{N}$

$$
t_{k+1}=\inf \left\{t:\|e(t)\| \geq \sigma\left(\left\|y_{t}\right\|\right) \wedge t>t_{k}\right\} .
$$

The three design parameters play important roles in the selection of the samples. The parameter $t_{0}$ specifies the amount of time in which the sampling mechanism is disabled at the beginning of the execution. This guarantees that $\sigma\left(\left\|y_{t}\right\|\right)>0$ immediately after $t_{0}$, which assures the existence of the first sampling time. Note that small values of $t_{0}$ can lead to small inter-event times at the beginning of the execution, since the output of the system may be still small at $t_{0}$. The parameter $h$ also prevents the system from having Zeno phenomenon as it will be proved in this section. 


\section{B. Stability analysis of nonlinear systems}

Definition 1: The event-triggered control system (3) with (7) and (8) is

- stable if $\forall \varepsilon>0$, there exists a $\delta=\delta(\varepsilon)>0$ such that $\|x(0)\| \leq \delta$ implies $\|x(t)\| \leq \varepsilon$ for all $t \geq 0$;

- asymptotically stable if it is stable and there exists a $\delta_{a}>0$ such that for any $\eta_{a}>0$ there exists a $T=$ $T\left(\delta_{a}, \eta_{a}\right)$ such that $\|x(0)\| \leq \delta_{a}$ implies $\|x(t)\| \leq \eta_{a}$ for all $t \geq T$;

- exponentially stable with decay rate $\gamma$ if there exist $\delta_{e}>$ 0 and $\eta_{e}>0$ such that $\|x(0)\| \leq \delta_{e}$ implies $\|x(t)\| \leq$ $\eta_{e} e^{-\gamma t}\|x(0)\|$ for all $t \geq 0$;

- globally asymptotically (respectively exponentially) stable if $\delta_{a}$ (respectively $\delta_{e}$, where $\eta_{e}$ is independent of $\delta_{e}$ ) can be an arbitrarily large, finite number.

Proposition 1: If there exists a continuous, nondecreasing function $\rho(s)>s$ such that the function $w$ defined by

$$
w: s \mapsto \alpha(s)-\beta\left(\sigma\left(\xi\left(\underline{\alpha}^{-1}(\rho(\bar{\alpha}(s)))\right)\right)\right)
$$

is of $\mathcal{K}$-class, then the event-triggered control system (3) with (7) and (8) is globally asymptotically stable for any finite parameters $h>0$ and $t_{0}>0$.

Roughly speaking, the $\mathcal{K}$-class property of function (9) means that the continuous control system is stable enough (represented by the first term in (9)) such that the effect of samplings (the second term) does not destabilize the system.

Proposition 2: For a given $\delta>0$, assume there exist scalars $\lambda_{1}>\lambda_{2}>0$ such that

$$
\begin{gathered}
\lambda_{1} s \leq \alpha\left(\bar{\alpha}^{-1}(s)\right), \\
\lambda_{2} s \geq \beta\left(\sigma\left(\xi\left(\underline{\alpha}^{-1}(s)\right)\right)\right)
\end{gathered}
$$

for all $0 \leq s \leq \delta$, then the event-triggered control system (3) with (7) and (8) is exponentially stable with decay rate $\gamma>0$ given as the unique solution of

$$
\gamma=\lambda_{1}-\lambda_{2} e^{\gamma h}
$$

In addition, if (10) holds for all $s \in[0, \infty)$, then the system is globally exponentially stable.

Remark 1: Note that big values of $\delta$ in Proposition 2 may lead to small values of $\lambda_{1}$ and big values of $\lambda_{2}$, in order to satisfy (10), which implies small values of the decay rate. On the other hand, from a simple inspection of Equation (11), it follows that the decay rate decreases when the parameter $h$ increases. In addition, $\lambda_{1}$ is the supremum of $\gamma$, that is $\lim _{h \rightarrow 0} \gamma(h)=\lambda_{1}-\lambda_{2}$, where $\gamma(h)$ is the solution of (11) as a function of $h$.

It is well-known that if the event-triggered mechanism is not selected appropriately then it may cause Zeno phenomenon in which the system generates an infinite number of samplings in a finite time. The next result guarantees that the control system provided with the proposed sampling algorithm is free of accumulations points, that is, Zeno solutions are not possible.

Proposition 3: For every $\delta>0$ and $T>0$, for any solution of the event-triggered control system (3) with (7) and (8), starting from an initial condition $x(0) \neq 0$ with $\|x(0)\| \leq \delta$, there is a finite sequence of triggering times in the interval $[0, T]$.

\section{APPLYING AND IMPROVING THE RESULTS}

\section{A. Application of previous results to LTI systems}

In this section, the closed-loop system under study is formed by an LTI plant given by

$$
\mathcal{P}:\left\{\begin{array}{l}
\dot{x}_{p}(t)=A_{p} x_{p}(t)+B_{p} u_{p}(t), \\
y_{p}(t)=C_{p} x_{p}(t)
\end{array}\right.
$$

and an LTI controller described as follows:

$$
\mathcal{C}:\left\{\begin{array}{l}
\dot{x}_{c}(t)=A_{c} x_{c}(t)+B_{c} u_{c}(t), \\
y_{c}(t)=C_{c} x_{c}(t)
\end{array}\right.
$$

where $A_{p}, B_{p}, C_{p}, A_{c}, B_{c}$, and $C_{c}$ are matrices of appropriate dimension. The dynamics of the event-triggered closedloop system can be described as

$$
\left\{\begin{array}{l}
\dot{x}(t)=(A+B C) x(t)+B e(t), \\
y(t)=C x(t)
\end{array}\right.
$$

for all $t \in\left[t_{k}, t_{k+1}\right)$ where $e(t)=y\left(t_{k}\right)-y(t)$ and

$$
A=\left[\begin{array}{cc}
A_{p} & B_{p} C_{c} \\
0 & A_{c}
\end{array}\right], \quad B=\left[\begin{array}{c}
0 \\
B_{c}
\end{array}\right], \quad C=\left[\begin{array}{cc}
C_{p} & 0
\end{array}\right] \text {. }
$$

For the analysis of the above system, the general Assumptions 1 and 2 are replaced by their counterparts for LTI systems

Assumption 3: The pair $(A, C)$ is observable.

Assumption 4: The controller $\mathcal{C}$ renders the system (14) with $e(t)=0$ for all $t \geq 0$ asymptotically stable, and thus, for every matrix $Q \in \mathbb{S}_{+}^{n}$ there exists a matrix $P \in \mathbb{S}_{+}^{n}$ such that

$$
(A+B C)^{\top} P+P(A+B C)=-Q .
$$

Henceforth, the function $\sigma \in \mathcal{K}$ for the sampling algorithm (8) will be given by $\sigma(s)=\sigma_{c} s$ with $\sigma_{c}>0$.

Proposition 4: Consider matrices $Q \in \mathbb{S}_{+}^{n}$ and $P \in \mathbb{S}_{+}^{n}$ satisfying (16) and $Q-I>0$. In addition, let $\sigma_{c}$ in the sampling algorithm (8) satisfy

$$
0<\sigma_{c}<\sqrt{\frac{\lambda_{m}(Q-I) \lambda_{m}(P)}{\left\|B^{\top} P P B\right\|\|C\|^{2} \lambda_{M}(P)}},
$$

then the event-triggered control system (14) with (7) and (8) is globally exponentially stable with decay rate $\gamma>0$ given as the unique solution of

$$
\gamma=\frac{\lambda_{m}(Q-I)}{\lambda_{M}(P)}-\frac{\left\|B^{\top} P P B\right\|\|C\|^{2} \sigma_{c}^{2}}{\lambda_{m}(P)} e^{\gamma h} .
$$

\section{B. Improving the results}

The previous result gives a bound on the parameter $\sigma_{c}$ in such way that exponential stability of the event-triggering implementation of an asymptotic stable control system is guaranteed. In addition, it provides information on the decay rate as a function of the parameter $h$. However, the equations for both $\sigma_{c}$ and $\gamma$ are coupled, and also nonlinear with respect to the matrix $P$. This makes it difficult to run an optimization problem, and in addition, to check whether the 
system is exponentially stable for a given setup of the design parameters $\sigma_{c}$ and $h$. The next result aims at overcoming these problems.

Proposition 5: For given scalars $\gamma, h, \sigma_{c}>0$, if there exist matrices $P \in \mathbb{S}_{+}^{n}, X \in \mathbb{S}^{n}, Y \in \mathbb{R}^{n \times n_{y}}, Z \in \mathbb{S}^{n_{y} \times n_{y}}$, and $U \in \mathbb{S}_{+}^{n_{y} \times n_{y}}$ such that

$$
\begin{gathered}
C^{\top} C-P<0 \\
{\left[\begin{array}{cc}
X & Y \\
\star & Z
\end{array}\right] \geq 0,} \\
\Psi:=\left[\begin{array}{cc}
X-Q & Y-P B \\
\star & Z-U
\end{array}\right]<-\left[\begin{array}{cc}
\left(\gamma+\sigma_{c}^{2}\|U\| e^{\gamma h}\right) P & 0 \\
0 & 0
\end{array}\right]
\end{gathered}
$$

hold, where $Q$ is given by (16), then the event-triggered control system given by (14), (7), and (8) is globally exponentially stable with decay rate $\gamma$.

Remark 2: Note that for a given $\gamma, \sigma_{c}$, and $h$, condition (21) is in the form of bilinear matrix inequality (BMI), for which it is usually hard to get a global solution. However, since $\lambda_{1}=\gamma+\sigma_{c}^{2}\|U\| e^{\gamma h}$ condition (21) can be rewritten as follows:

$$
\Psi<-\left[\begin{array}{cc}
\lambda_{1} P & 0 \\
0 & 0
\end{array}\right],
$$

which is an LMI for a given $\lambda_{1}$ and it can be solved by some LMI Toolbox. In addition, the convexity with respect to $\lambda_{1}$ guarantees that if (22) holds for some $\hat{\lambda}_{1}$ then it also hold for all $\lambda_{1} \in\left[0, \hat{\lambda}_{1}\right]$. Therefore, it is possible to find the maximum $\lambda_{1}$ by a simple line search, with stop condition defined by the feasibility of the LMIs. Finally, by minimizing $\|U\|$ for every $\lambda_{1}$, we maximize the allowed $\sigma_{c}$ for this value of $\lambda_{1}$. This indirectly increases the interval between two samples.

\section{EXAMPLES}

\section{A. Nonlinear example}

Consider an output feedback control system (3) where the plant is defined by the following functions

$$
f_{p}\left(x_{p}\right)=\left[\begin{array}{c}
-x_{1}-x_{2} x_{3}+x_{2}+u_{p} \\
x_{1} x_{3}-10 x_{2}-x_{3} \\
x_{1} x_{2}+x_{2}-x_{3}^{3}
\end{array}\right], \quad g_{p}\left(x_{p}\right)=x_{1}
$$

with $x_{p}=\left[\begin{array}{lll}x_{1} & x_{2} & x_{3}\end{array}\right]^{\top}$, and the controller by

$$
f_{c}\left(x_{c}\right)=-10 x_{c}-u_{c}, \quad g_{c}\left(x_{c}\right)=x_{c} .
$$

The distinguishability Assumption 1 of the open-loop system $(\mathcal{C}, \mathcal{P})$ can be checked by computing the Lie derivative up to third-order (see [20]).

An ISS Lyapunov function for the closed-loop system can be found by using SOStools [16]. The function V with three significant digits is given by

$$
\begin{aligned}
V(x) & =0.706 x_{1}^{4}+0.1429 x_{2}^{4}+0.118 x_{3}^{4}+0.9781 x_{c}^{4} \\
& +0.117 x_{1}^{3} x_{2}+0.372 x_{1}^{2} x_{2}^{2}+0.437 x_{1}^{2} x_{3}^{2}+0.975 x_{1}^{2} x_{c}^{2} \\
& -0.05 x_{1} x_{c}^{3}+0.214 x_{2}^{2} x_{3}^{2}+0.1598 x_{2}^{2} x_{c}^{2}-0.002 x_{2} x_{3}^{3} \\
& -0.005 x_{2} x_{c}^{3}+0.202 x_{3}^{2} x_{c}^{2}+0.37 x_{3} x_{c}^{3}+0.176 x_{1} x_{2} x_{c}^{2} \\
& +0.004 x_{2} x_{3} x_{c}^{2}
\end{aligned}
$$

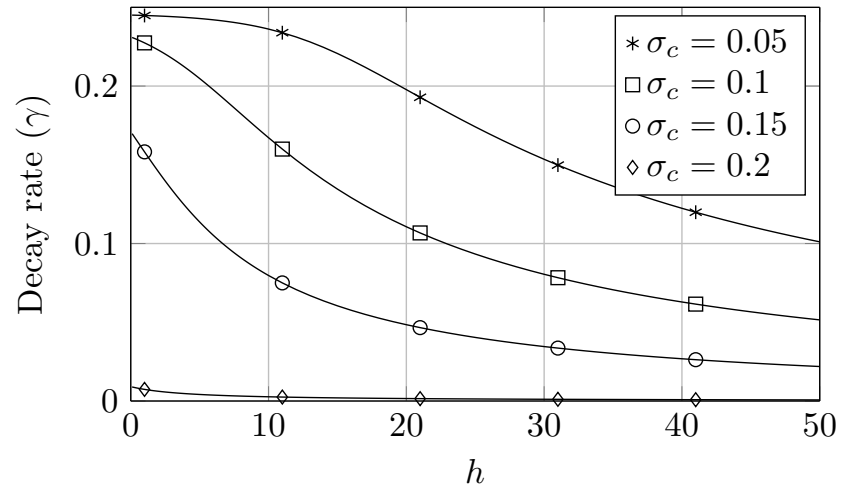

Fig. 1. Decay rate $\gamma$ vs. $h$ for several values of the parameter $\sigma_{c}$ for the Example V-A.

\begin{tabular}{ccccc}
\hline$\sigma_{c} \backslash$ & 0.1 & 0.5 & 1 & 10 \\
\hline 0.05 & $0.0027(0.20)$ & $0.0086(0.27)$ & $0.0086(0.34)$ & $0.0090(1.04)$ \\
0.1 & $0.0054(0.39)$ & $0.0223(0.53)$ & $0.0223(0.67)$ & $0.0230(1.96)$ \\
0.2 & $0.0106(0.75)$ & $0.0341(1.01)$ & $0.0884(1.27)$ & $0.1290(3.63)$ \\
\hline
\end{tabular}

TABLE I

MiNimUM INTER-EVENT TIME (AVERAGE OF THE INTER-EVENT TIME IN BRACKETS) FOR 100 RANDOM EXECUTIONS OF EXAMPLE V-A FOR A SIMULATION TIME OF 40s.

for all $x=\left[\begin{array}{llll}x_{1} & x_{2} & x_{3} & x_{c}\end{array}\right]^{\top} \in \mathbb{R}^{4}$. The $\mathcal{K}$-class functions of Assumption 2 are also found by SOStools, and are given by

$$
\begin{aligned}
\alpha & (s)=0.0768 s^{4}, & \bar{\alpha}(s) & =1.1667 s^{4}, \\
\alpha(s) & =0.28716 s^{4}, & \beta(s) & =1.0067 s^{4}
\end{aligned}
$$

for all $s \geq 0$. The function $\sigma$ of the sampling algorithm (8) is simply defined by $\sigma(s)=\sigma_{c} s$ with $\sigma_{c}>0$, then the selection of $\lambda_{1}=0.246$ and $\lambda_{2}=148.04 \sigma_{c}^{4}$ guarantees that condition (10) holds. Therefore, if $\sigma_{c} \leq 0.201$, the global exponential stability of the event-triggered implementation of the control system follows from Proposition 2. The decay rate $\gamma$ is obtained by solving the equation $\gamma=\lambda_{1}-\lambda_{2} e^{\gamma h}$ for a given $h>0$. Fig. 1 shows how the parameter $h$ affects the decay rate $\gamma$ of the system for several values of $\sigma_{c}$. Note that big values of $h$ and $\sigma_{c}$ lead to a small decay rate. Moreover, it can be seen that the parameter $\sigma_{c}$ has the greatest impact on $\gamma$. Table I provides the minimum and the average of the interevent times of the 100 executions of the system with random initial conditions, a simulation time of 40 s, and several values of the design parameters (the parameter $t_{0}=0.1$ has been used). It can be observed that an increment on both $\sigma_{c}$ and $h$ leads to an increment of the inter-event times. This effect is more evident in the average of the inter-event times. 


\section{B. LTI example}

In this example, we consider the control system studied in [7], where the plant and the controller are given by

$$
\begin{aligned}
& \mathcal{P}:\left\{\begin{array}{l}
\dot{x}_{p}(t)=\left[\begin{array}{cc}
0 & 1 \\
-2 & 3
\end{array}\right] x_{p}(t)+\left[\begin{array}{l}
0 \\
1
\end{array}\right] u_{p}(t), \\
y(t)=\left[\begin{array}{ll}
-1 & 4
\end{array}\right] x_{p}(t) .
\end{array}\right. \\
& \mathcal{C}:\left\{\begin{array}{l}
\dot{x}_{c}(t)=\left[\begin{array}{cc}
0 & 1 \\
0 & -5
\end{array}\right] x_{c}(t)+\left[\begin{array}{l}
0 \\
1
\end{array}\right] u_{c}(t), \\
u_{c}(t)=\left[\begin{array}{ll}
1 & -4
\end{array}\right] x_{c}(t) .
\end{array}\right.
\end{aligned}
$$

Assumptions 3 and 4 hold for the closed-loop system, and thus Propositions 4 and 5 can be applied. Following Remark 2, a set of values of $\lambda_{1}$ and $U$ has been computed by Proposition 5. From these data, it follows that the eventtriggered implementation is exponentially stable if $\sigma_{c} \leq$ 0.0351. In addition, the supremum of the decay rate is 0.97 . In Fig. 2, the effects of the parameters $\sigma_{c}$ and $h$ on the decay rate $\gamma$ are shown. The results show that the decay rate decreases to zero as $\sigma_{c}$ approaches its maximum. In addition, the decay rate is also decreased by increasing $h$. Note that for small values of $h$ (approximately $h \leq 1$ ) the changes on the decay rate are small.

In addition, 100 executions of the system have been run with random initial conditions, a simulation time of $20 \mathrm{~s}$, $\sigma_{c}=0.035$ (close to its maximum) and $t_{0}=0.1$. Table II shows the minimum and the average of the inter-event times for several values of $h$. As in the previous example, it can be observed that both $\sigma_{c}$ and $h$ lead to an increase of the inter-event times.

On the other hand, the results in [7] show that practical stability can be guaranteed by considering the sampling algorithm

$$
t_{k+1}=\inf \left\{t:\|e(t)\|^{2} \geq \sigma_{p}\|y(t)\|^{2}+\varepsilon \wedge t>t_{k}\right\} .
$$

The solutions converge to a set that depends on the parameter $\varepsilon>0$. In addition, by adding the term $\varepsilon$ the existence of Zeno solutions is avoided as proved in [7]. In order to compare the proposed algorithm with (29), we consider the execution of the system with both algorithms and the initial condition $x(0)=\left[\begin{array}{lll}12.5 & -12.5-12.5 & 12.5\end{array}\right]^{\top}$. The parameters for the sampling algorithm (29) are $\varepsilon=0.001$ and $\sigma_{p}=0.001$, and for the proposed algorithm (8) we set the parameters as follows: $t_{0}=0.1, \sigma_{c}=0.035$, and $h=3$. Note that $\sigma_{c}$ is almost the square root of $\sigma_{p}$. Thus the algorithms (29) and (8) are comparable on this aspect. Fig. 3 shows the norm of the state $x$ as a function of the time. It can be seen that both algorithms provide a similar decay rate. However, the proposed algorithm guarantees the asymptotic stability while only practical stability is guaranteed by algorithm (29). The inter-event times are shown in Fig. 4. Note how the proposed algorithm increases the inter-event time during the transient state. However, the inter-event times are slightly smaller during the steady state. Finally, Fig. 5 shows the density of the inter-event time. It can be observed that the inter-event times are condensed around 0.1s for the proposed algorithm.

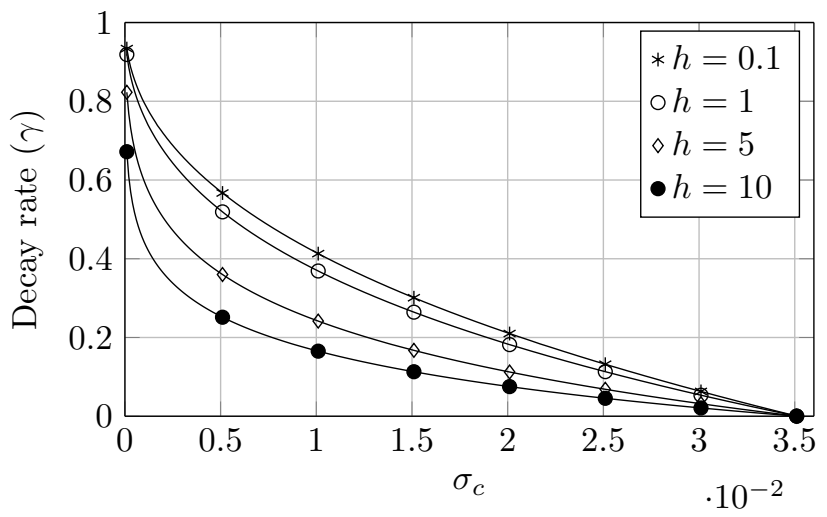

Fig. 2. Decay rate $\gamma$ vs. $\sigma_{c}$ for several values of the parameter $h$ for the Example V-B.

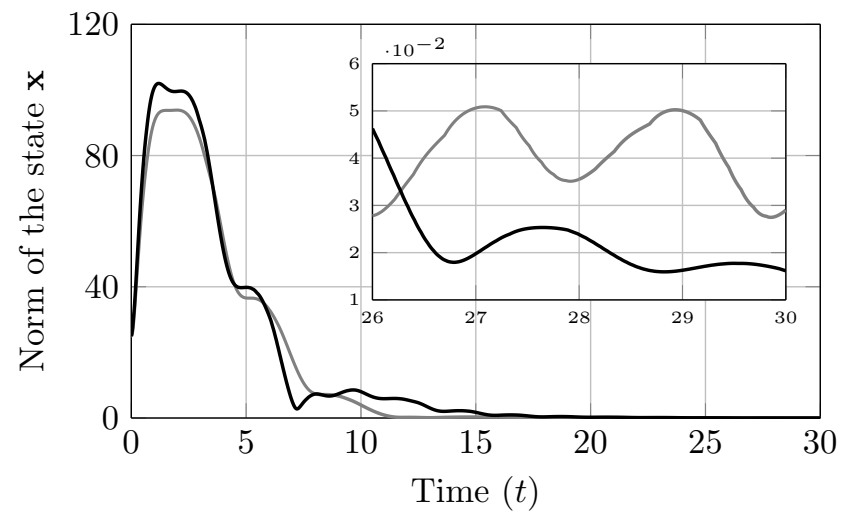

Fig. 3. Norm of the state $x$ of the system in Example V-B with initial condition $x(0)=[12.5,-12.5,-12.5,12.5]^{\top}$ : sampling algorithm (29) with $\varepsilon=0.001$ and $\sigma_{p}=0.001$ (grey line), proposed sampling algorithm (8) with $t_{0}=0.1, \sigma_{c}=0.035$, and $h=3$ (black line). Close-up of the norm at $t \in[26,30]$ (inserted figure).

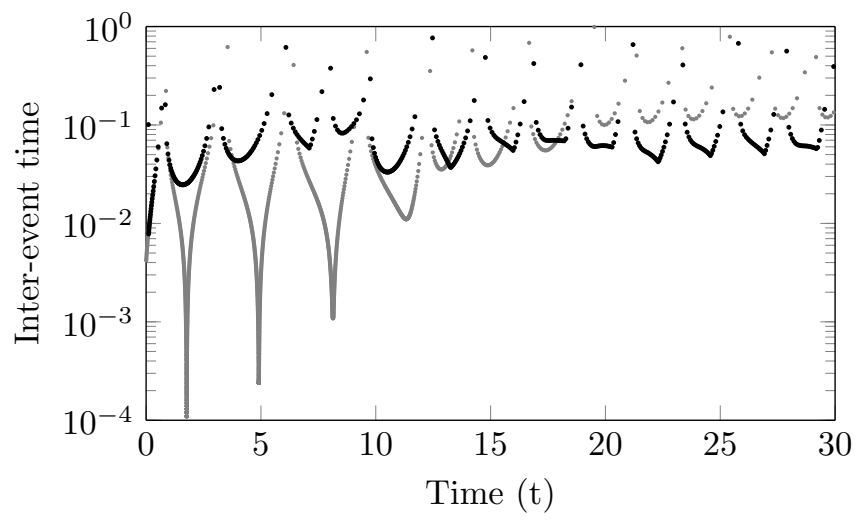

Fig. 4. Inter-event times for Example V-B with initial condition $x(0)=$ $[12.5,-12.5,-12.5,12.5]^{\top}$ : sampling algorithm (29) with $\varepsilon=0.001$ and $\sigma_{p}=0.001$ (grey points), proposed sampling algorithm (8) with $t_{0}=0.1$, $\sigma_{c}=0.035$, and $h=3$ (black points). 


\begin{tabular}{cccc}
\hline$\sigma_{c} h$ & 0.1 & 1 & 5 \\
\hline 0.02 & $0.8 \cdot 10^{-3}(0.012)$ & $0.8 \cdot 10^{-3}(0.0304)$ & $0.8 \cdot 10^{-3}(0.0495)$ \\
0.035 & $0.6 \cdot 10^{-3}(0.0198)$ & $1 \cdot 10^{-3}(0.0450)$ & $1 \cdot 10^{-3}(0.0729)$ \\
\hline
\end{tabular}

TABLE II

Minimum INTER-EVENT TIME (AVERAGE OF THE INTER-EVENT TIME IN BRACKETS) FOR 100 RANDOM EXECUTIONS OF EXAMPLE V-B FOR A SIMULATION TIME OF $20 \mathrm{~s}$.
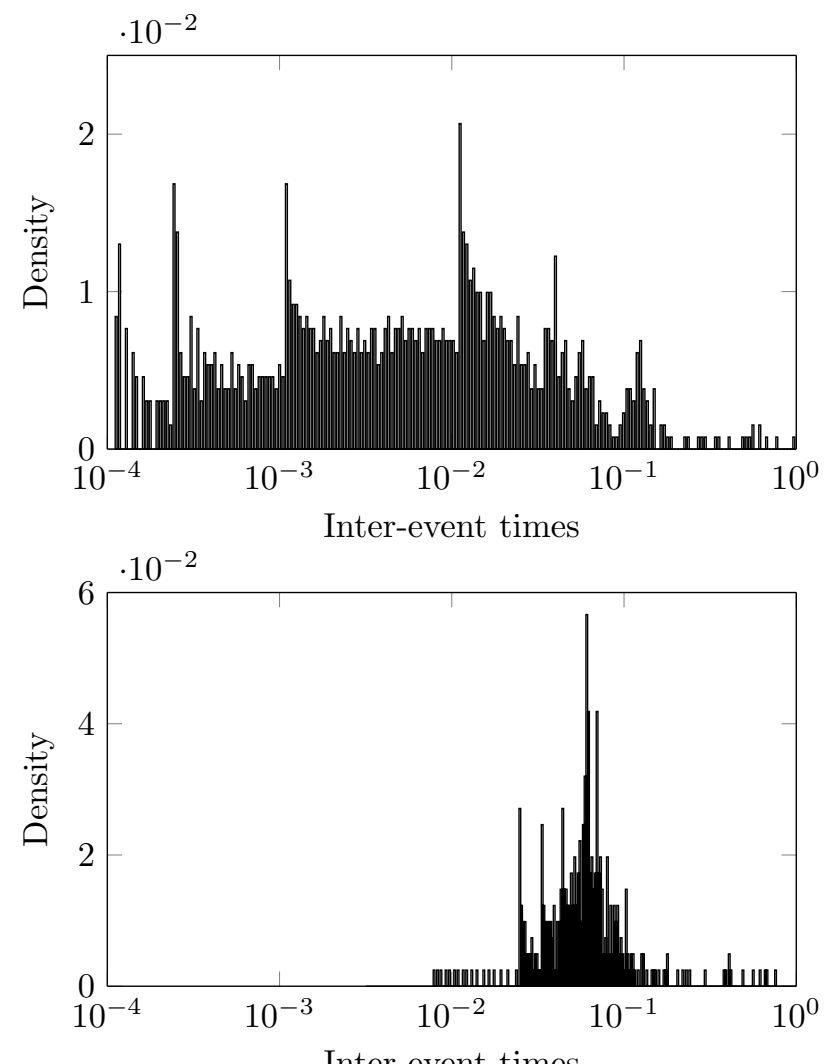

Inter-event times

Fig. 5. Density function of the inter-event time for Example V-B with initial condition $x(0)=[12.5,-12.5,-12.5,12.5]^{\top}$ and a simulation time of 30s: sampling algorithm (29) with $\varepsilon=0.001$ and $\sigma_{p}=0.001$ (top figure), proposed sampling algorithm (8) with $t_{0}=0.1, \sigma_{c}=0.035$, and $h=3$ (bottom figure).

\section{CONCLUSIONS}

An event-triggering algorithm has been proposed to guarantee the asymptotic and exponential stability of the eventtriggered implementation of an output-based control system. The novelty of the algorithm comes from the use of a sampling condition based on the history of the output. Under this event-triggering scheme, it is proved that Zeno solutions are avoided. Both nonlinear and linear systems were considered. For LTI systems a criterion for exponential stability has been provided in the form of LMI. Several simulation examples show how the inter-event times can be increased by a suitable design parameters, but at the price of reducing the decay rate of convergence of the trajectories. For the future work, it could be interesting to consider a sampling mechanism for the controller output, as well as to extend the results to the tracking control problem, for instance considering known references and analyzing the system in the error space.

\section{REFERENCES}

[1] M. Abdelrahim, R. Postoyan, J. Daafouz, and D. Nesic. Stabilization of nonlinear systems using event-triggered output feedback controllers. IEEE Transactions on Automatic Control, 61(9):2682-2687, 2016.

[2] A. Adaldo, F. Alderisio, D. Liuzza, G. Shi, D. V. Dimarogonas, M. di Bernardo, and K. H. Johansson. Event-triggered pinning control of complex networks with switching topologies. In IEEE 53rd Conference on Decision and Control, pages 2783-2788, Los Angeles (CA), USA, 2014.

[3] A. Adaldo, F. Alderisio, D. Liuzza, G. Shi, D. V. Dimarogonas, M. di Bernardo, and K. H. Johansson. Event-triggered pinning control of switching networks. IEEE Trans. on Control of Network Systems, 2(2):204-213, 2015.

[4] V. Andrieu. Convergence speed of nonlinear Luenberger observers. SIAM J. Control and Optimization, 52(5):2831-2856, 2014.

[5] K. Åström. Event based control. In Analysis and Design of Nonlinear Control Systems, pages 127-148. Springer, 2008.

[6] Z. Bartosiewicz. Local observability of nonlinear systems. Systems \& Control Letters, 25:295-298, 1995.

[7] M.C.F. Donkers and W.P.M.H. Heemels. Output-based eventtriggered control with guaranteed $L_{\infty}$-gain and improved and decentralised event-triggering. IEEE Transactions on Automatic Control, 57(6):1362-1376, 2012.

[8] E. Fridman. Introduction to Time-Delay Systems. Systems \& Control: Foundations \& Applications. Birkhäuser, 2014.

[9] A. Halanay. Differential equation: stability, oscillations, time lags. Academic, New York, 1966.

[10] W.P.M.H. Heemels, M.C.F. Donkers, and A.R. Teel. Periodic eventtriggered control for linear systems. IEEE Transactions on Automatic Control, 58:847-861, 2013.

[11] W.P.M.H. Heemels, K.H. Johansson, and P. Tabuada. An introduction to event-triggered and self-triggered control. In Proc. 51st IEEE Conf. Decision \& Control, pages 3270-3285, Maui, HI, 2012.

[12] W.P.M.H. Heemels, R. Postoyan, M.C.F. Donkers, A.R. Teel, A. Anta, P. Tabuada, and D. Nesic. Periodic event-triggered control. In M. Miskowicz, editor, Event-based control and signal processing. CRC Press/Taylor \& Francis, 2015.

[13] K. H. Johansson, M. Egerstedt, J. Lygeros, and S. Sastry. On the regularization of zeno hybrid automata. Systems \& Control Letters, 38(3):141-150, 1999.

[14] J. Lunze and D. Lehmann. A state-feedback approach to event-based control. Automatica, 46(1):211-215, 2010.

[15] M. Miskowicz. Event-based control and signal processing. CRC Press/Taylor \& Francis Group, 2015.

[16] A. Papachristodoulou, J. Anderson, G. Valmorbida, S. Prajna, P. Seiler, and P. A. Parrilo. SOSTOOLS: Sum of squares optimization toolbox for MATLAB. http://arxiv.org/abs/1310.4716, 2013.

[17] C. Peng and Q.L. Han. Output-based event-triggered $H_{\infty}$ control for sampled-data control systems with nonuniform sampling. In American Control Conf., pages 1727-1732, Washington, 2013.

[18] R. Postoyan, P. Tabuada, D. Nesic, and A. Anta. A framework for the event-triggered stabilization of nonlinear systems. IEEE Transactions on Automatic Control, 60(4):982-996, 2015.

[19] A. Seuret, C. Prieur, and N. Marchand. Stability of non-linear systems by means of event-triggered sampling algorithms. IMA Journal of Mathematical Control and Information, 31(3):415-433, 2014

[20] E. D. Sontag. A concept of local observability. Systems \& Control Letters, 5:41-47, 1984.

[21] P. Tabuada. Event-triggered real-time scheduling of stabilizing control tasks. IEEE Transactions on Automatic Control, 52(9):1680-1685, 2007.

[22] A. Tanwani, C. Prieur, and M. Fiacchini. Observer-based feedback stabilization of linear systems with event-triggered sampling and dynamic quantization. Systems \& Control Letters, 94:46-56, 2016.

[23] A. Tanwani, A.R. Teel, and C. Prieur. On using norm estimators for event-triggered control with dynamic output feedback. In Proc. 54th IEEE Conf. on Decision and Control, pages 5500-5505, Osaka, Japan, 2015. 